\title{
RELATIVE ORIENTATION OF VLBI CELESTIAL REFERENCE FRAMES
}

\author{
E.F. Arias, J.- F. Lestrade \\ Bureau des Longitudes \\ 77 Av. Denfert - Rochereau, 75014 Paris, France \\ M. Feissel \\ Bureau International de l'Heure \\ Observatoire de Paris \\ 61 Av. de l'Observatoire, 75014 Paris, France
}

\begin{abstract}
Several series of celestial reference frames have been produced during the past few years as part of VLBI geodynamic programs. They consist in coordinates of 20 to 150 extragalactic radio sources with a precision at the level of $0.001^{\prime \prime}$. The relative orientations of these frames and the evolution of each series of catalogs are studied.
\end{abstract}

\section{Introduction}

During the last ten years, three groups, at Jet Propulsion Laboratory (JPL) on the one hand, at Goddard Space Flight Center (GSFC) and National Geodetic Survey (NGS) on the other hand have published catalogues of precise positions of extragalactic radio sources derived from Very Long Baseline Interferometry (VLBI) observations. The JPL and GSFC/NGS groups have conducted independent observation campaigns with different VLBI networks and data acquisition systems. They used independent physical models for the VLBI observables and different software for the data analysis. Consequently, the question arises of the consistency of the VLBI celestial frames associated with these catalogues.

We have compared the five VLBI celestial reference frames summarized in Table I to determine their relative orientations. We have used the most recent catalogues produced by JPL and GSFC and those in use in the Earth Rotation programs. RSC (JPL) 83 R 05 is the catalogue used so far by the JPL Earth Rotation program TEMPO and RSC (NGS) $86 \mathrm{R} 01$ is the one used by IRIS (International Radio Interferometric Surveying).

The estimation of the relative orientations of these celestial frames is useful for intercomparing independent Earth Rotation Parameter (ERP) series measured with the VLBI technique. Each series is referred to specific celestial and terrestrial reference frames adopted or solved for in the data reduction procedure. Hence, systematic differences in the pole coordinates $(\Delta x, \Delta y)$ and the Universal Time $\left(\Delta I^{i} T 1\right)$ are induced by the three rotations $A_{1}, A_{2}$ and $A_{3}$ between two celestial reference frames and the three rotations $R_{1}, R_{2}$ and $R_{3}$ between two terrestrial reference frames. These systematic differences are given by Zhu and Mueller (1983):

$$
\begin{aligned}
& \Delta x=R_{2}+A_{1} \sin t-A_{2} \cos t \\
& \Delta y=R_{1}-A_{1} \cos t-A_{2} \sin t \\
& \text { f. } \Delta U T 1=-R_{3}+A_{3}
\end{aligned}
$$


where $\mathrm{t}$ is the sidereal time and $\mathrm{f}$ is the conversion factor between UT1 and sidereal time. The three rotations $R_{1}, R_{2}$ and $R_{3}$ (as well as translations and scaling factors) between the corresponding terrestrial reference frames and series of ERP can be found in the Annual Reports of the Bureau International de l'Heure. In this paper, we provide the three rotations $A_{1}, A_{2}$ and $A_{3}$ for the five VLBI celestial reference frames summarized in Table I.

TABLE I

VLBI Celestial references frames compared. The column $\mathrm{N}$ provides the number of radio sources in each catalogue; the column $\mathrm{N}_{1}$ provides the number of radio sources in the IRIS and JPL Earth Rotation Programs.

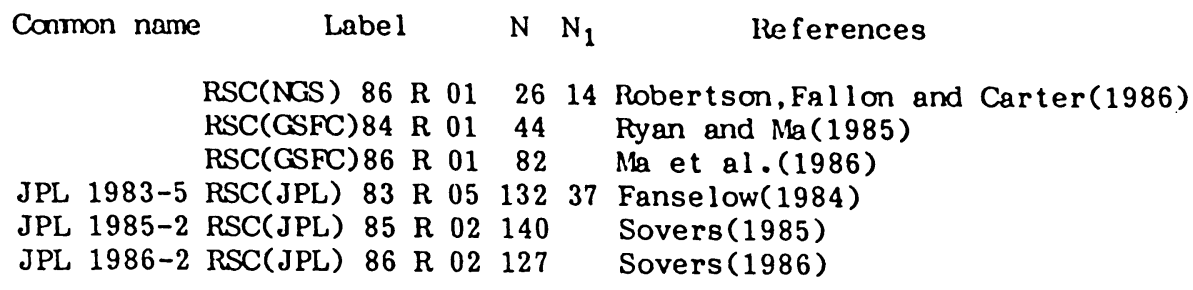

\section{Transformation Matrix}

The relative orientation between two celestial reference frames is obtained by computing three differential rotations around the coordinate axes. A source common to both frames and of direction $\vec{d}$ has two sets of coordinates, primed coordinates in catalogue 1 and doubleprimed ones in catalogue 2. In vectorial form, the transformation between these two celestial reference frames can be written as:

$$
\left(\begin{array}{l}
d_{x}^{\prime}-d_{x}^{\prime \prime} \\
d_{y}^{\prime \prime}-d_{y}^{\prime \prime} \\
d_{z}^{\prime \prime}-d_{z}^{\prime \prime}
\end{array}\right)=[M]\left(\begin{array}{l}
d_{x}^{\prime \prime} \\
d_{y}^{\prime \prime} \\
d_{z}^{\prime \prime}
\end{array}\right)
$$

where the rotation matrix $[\mathrm{M}]$ is:

$$
[M]=\left(\begin{array}{ccc}
0 & A_{3} & -A_{2} \\
-A_{3} & 0 & A_{1} \\
A_{2} & -A_{1} & 0
\end{array}\right)
$$

$A_{1}, A_{2}$ and $A_{3}$ being the differential rotations around the $\mathrm{x}, \mathrm{y}$ and $\mathrm{z}$ axes, respectively, in the sense frame 2 towards frame 1 . 
The differences between the equatorial coordinates of a source common to the two catalogues are to first order with respect to $A_{1}, A_{2}$ and $A_{3}$ :

$$
\begin{aligned}
\cos \delta \Delta \alpha & =\left(\alpha^{\prime}-\alpha^{\prime \prime}\right) \cos \delta=A_{1} \sin \delta \cos \alpha+A_{2} \sin \delta \sin \alpha-A_{3} \cos \delta \\
\Delta \delta & =\delta^{\prime}-\delta^{\prime \prime}=-A_{1} \sin \alpha+A_{2} \cos \alpha
\end{aligned}
$$

For $\mathrm{N}$ sources common to the two catalogues, a system of $2 \mathrm{~N}-1$ equations can be formed, since the right ascension of the source $3 \mathrm{C} 273$ is fixed to an a priori value in the catalogues. The three unknowns $A_{1}, A_{2}, A_{3}$ can be determined by a weighted least squares fit. The formal errors $\sigma_{\alpha^{\prime}}, \sigma_{\delta^{\prime}}, \sigma_{\alpha^{\prime \prime}}, \sigma_{\delta^{\prime \prime}}$ for the source positions provided in the catalogues are used to compute the uncertainty of the positions differences $(\Delta \alpha, \Delta \delta)$ according to:

$$
\sigma_{\Delta \alpha}=\sqrt{\sigma_{\alpha^{\prime}}{ }^{2}+\sigma_{\alpha^{\prime \prime}}{ }^{2}}
$$

and analogously for $\sigma_{\Delta \delta}$.

\section{Error Analysis}

Realistic variances for the source coordinates in the two catalogues can be estimated by applying additive variance and/or a scaling factor to the formal errors. The two procedures have been tested for all pairs of catalogues, using the criterion that the normalized $\chi^{2}$ of the least squares solution of system (1) be close to unity. It was found that $\chi^{2}$ has little sensitivity to the scaling factors, and a high sensitivity to additive variances. Therefore, we have sought a normal distribution for the post-fit residuals by adding a constant $\mathrm{C}$ to the summed a priori variances of the position differences of the common sources, i.e., $\sigma_{\Delta \alpha}$ becomes:

$$
\sigma_{\Delta \alpha}=\sqrt{\sigma_{\alpha^{\prime}}{ }^{2}+\sigma_{\alpha^{\prime \prime}}{ }^{2}+C}
$$

and similarly for $\sigma_{\Delta \delta}$. In general, the application of the additive constant $\mathrm{C}$ results in an increase of $0.0005^{\prime \prime}$ to $0.0015^{\prime \prime}$ for $\sigma_{\Delta \alpha}$ and $\sigma_{\Delta \delta}$ ( $50 \%$ to $150 \%$ in relative value).

\section{Results}

We have computed the three rotations $A_{1}, A_{2}, A_{3}$ for two different sets of data:

a) the radio sources common to each pair of catalogues (results in Table II).

b) the 19 radio sources common to all the catalogues (results in Table III).

These two solutions are consistent. In general, the three rotations are slightly smaller in solution (b) than those in solution (a). In the two cases, $\left|A_{3}\right|$ is the largest angle.

As a verification of our calculation, the closure relations for the triplets of catalogues were formed. In principle, if $d_{x}, d_{y}, d_{z}$ are the coordinates of a source of direction $\vec{d}$, and if $M(12), M(23), M(31)$ are the transformation matrices from catalogue 1 to 2 , from 2 to 3 and from 3 to 1 , then the product of these three matrices should be the unit matrix.

For the solution (b) we have found that the closing errors are well within the mean error of the combined rotation uncertainties, except for three cases where the closing error 
Differential rotations $A_{1}, A_{2}, A_{3}$ in the sense frame 2 towards frame 1. The column $\mathrm{N}$ gives the number of sources carmon to each pair. Units : $0.001 "$.

$$
\text { Catalogue } 1 \text { - Catalogue } 2 \quad A_{1} \quad A_{2} \quad A_{3} \quad N
$$

RSC(NGS) $86 \mathrm{R} 01-\mathrm{RSC}(\mathrm{JPL}) 85 \mathrm{R} 02+0.58 \pm 0.4+0.45 \pm 0.4+1.91 \pm 0.321$

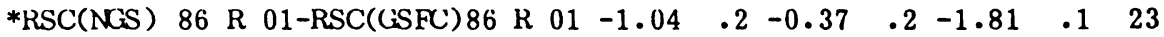

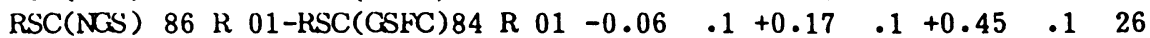

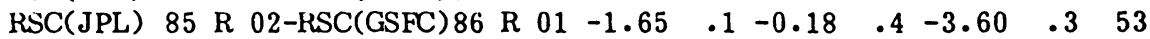

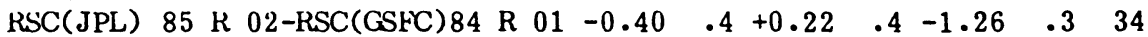

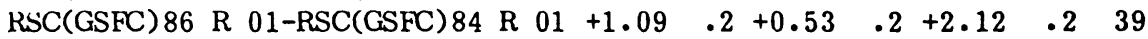

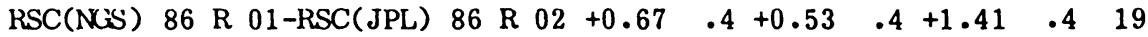

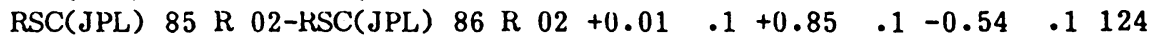

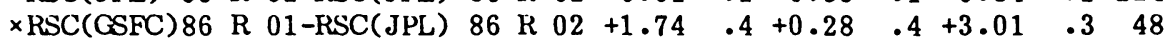

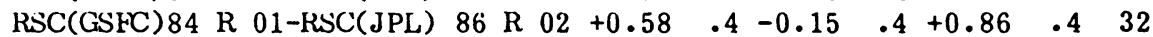

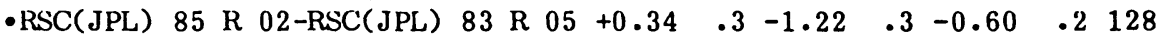

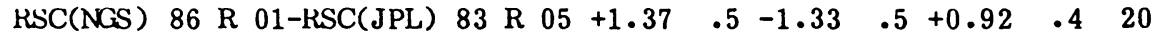

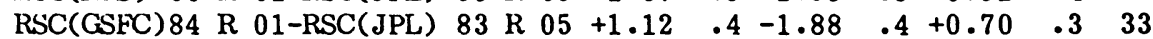

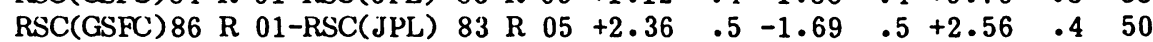

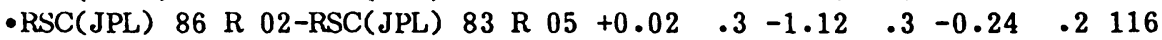

Radio sources deleted in the least squares solution :

* $1642+690,1803+784,2134+004$

$\times 1342+663$

- $0300+470,0454+844$

\section{TABLE III}

Differential rotations $A_{1}, A_{2}, A_{3}$ in the sense frame 2 towards frame 1. These rotations are calculated with the 19 sources common to all the catalogues. Units : $0.001 "$.

Catalogue 1 - Catalogue 2

RSC(NGS) $86 \mathrm{R} 01-\mathrm{RSC}$ (JPL) $85 \mathrm{~K} 02$ RSC(NGS) $86 \mathrm{R} \quad 01-\mathrm{RSC}(\mathrm{GSFC}) 86 \mathrm{R} 01$ RSC(NGS) $86 \mathrm{R} 01-\mathrm{RSC}(\mathrm{GSFC}) 84 \mathrm{R} 01$ $\mathrm{kSC}(\mathrm{JPL}) 85 \mathrm{R}$ 02-RSC(GSFC) $86 \mathrm{R} 01$ $\mathrm{RSC}(\mathrm{JPL}) 85 \mathrm{R} 02-\mathrm{RSC}$ (GSFC) $84 \mathrm{R} 01$ *RSC(GSFC) $86 \mathrm{~K}$ 01-RSC(GSFC) $84 \mathrm{k} 01$ RSC(NGS) $86 \mathrm{R} 01-\mathrm{RSC}(\mathrm{JPL}) 86 \mathrm{R} 02$ RSC(JPL) $85 \mathrm{R} 02-\mathrm{RSC}(\mathrm{JPL}) 86 \mathrm{R} 02$ $\mathrm{KSC}(\mathrm{GSHC}) 86 \mathrm{~K} 01-\mathrm{RSC}(\mathrm{JPL}) 86 \mathrm{R} 02$ $\mathrm{RSC}(\mathrm{GSFC}) 84 \mathrm{R}$ 01-RSC(JPL) $86 \mathrm{R} 02$ $\mathrm{RSC}(\mathrm{JPL}) 85 \mathrm{R}$ 02-RSC(JPL) $83 \mathrm{R} 05$ $\mathrm{RSC}(\mathrm{NGS}) 86 \mathrm{R} 01-\mathrm{RSC}(\mathrm{JPL}) 83 \mathrm{R} 05$ KSC(GSFC) $84 \mathrm{R} 01-\mathrm{KSC}(\mathrm{JPL}) 83 \mathrm{R} 05$ $\mathrm{RSC}(\mathrm{GSFC}) 86 \mathrm{R} \mathrm{01-RSC(JPL)} 83 \mathrm{R} 05$ RSC(JPL) 86 R 02-RSC(JPL) 83 R 05
$A_{1}$

$\begin{array}{llllll}+0.52 \pm 0.4 & +0.37 \pm 0.4 & +1.75 \pm 0.3 \\ -0.90 & .2 & -0.37 & .2 & -1.80 & .2 \\ +0.05 & .2 & +0.25 & .2 & +0.38 & .1 \\ -1.23 & .4 & -0.45 & .4 & -2.98 & .3 \\ -0.39 & .4 & -0.13 & .4 & -1.32 & .3 \\ +1.03 & .2 & +0.31 & .2 & +1.96 & .2 \\ +0.67 & .4 & +0.53 & .4 & +1.41 & .4 \\ -0.02 & .1 & +0.25 & .1 & -0.40 & .1 \\ +1.29 & .4 & +0.60 & .4 & +2.64 & .4 \\ +0.61 & .4 & +0.19 & .4 & +1.02 & .4 \\ +0.73 & .5 & -1.58 & .6 & -0.67 & .7 \\ +1.29 & .5 & -1.28 & .5 & +0.88 & .4 \\ +1.18 & .5 & -1.57 & .5 & +0.50 & .4 \\ +2.07 & .5 & -1.16 & .6 & +2.17 & .5 \\ +0.60 & .5 & -1.52 & .6 & -0.42 & .5\end{array}$

* Radio source 1253-055 was deleted in the least squares solution. 
in $A_{3}$ is at the $3 \sigma$ level. For the solution (a), the closing errors are well within the mean errors of the combined rotation uncertainties, except for three other cases where the closing error is at the $4 \sigma$ level in $A_{2}$. This level of agreement between the transformation matrices is thought satisfactory.

The differences for $A_{1}, A_{2}, A_{3}$ between solutions (a) and (b) in tables II and III are in general in agreement with the estimated uncertainties. This indicates that the latter are probably realistic.

The first line in Table II compares well with the rotations found for the same catalogues by Brosche et al (1986): $+0.3 \pm 0.7,+0.2 \pm 0.7,+1.6 \pm 0.5$, in units of $0.001^{\prime \prime}$.

\section{Discussion}

This study shows that the celestial reference frames realised by the programs at NGS, GSFC and JPL have their directions of axes consistent within $0.003^{\prime \prime}$. This is a remarkable agreement considering the diversity of observing strategies and data analysis.

The largest of the three rotation angles between pairs of catalogues is, in general, $A_{3}$. This may be partly due to the way the various VLBI networks resolve the complex source $3 \mathrm{C} 273$ used to fix arbitrarily the origin of the VLBI right ascensions in all catalogues.

The error analysis presented here is preliminary. It indicates that an additive variance corresponding roughly to $0.001^{\prime \prime}$ is necessary in the coordinate differences to obtain a normal distribution of the post-fit residuals. A refinement in the analysis could be to use two different additive constants $\mathrm{C}$ in $\alpha$ and $\delta$ (Arias et al, 1986)

The rotation angles estimated statistically with the common radio sources between catalogues have uncertainties in the range of $0.0001^{\prime \prime}$ to $0.0005^{\prime \prime}$. This range is confirmed by the stability of the estimation when the number of radio sources is changed. Consequently, the maintenance of a celestial reference system by means of VLBI coordinates of radio sources with a no-rotation condition could be ensured within $0.001^{\prime \prime}$.

\section{Acknowledgements}

The authors are indebted to C. Boucher and D.S. Robertson for stimulating discussions. J. L. Fanselow and O. J. Sovers kindly provided the three JPL catalogs. This study was partially funded by CNRS (UA 1125).

\section{References}

Arias, E.F., Feissel, M. and Lestrade J.-F. (1986). 'Intercomparison of VLBI Celestial Reference Frames' (in preparation).

Brosche, P., Sinachopoulos, D. (1986). 'Comparisons of Positions of Extragalactic Compact Radio Sources' (preprint).

Fanselow, J.L. (1984). RSC (JPL) 83 R 05 (private communication)

$\mathrm{Ma}$, C. et al.(1986). 'Radio Source Positions from VLBI'. Astron. J. (in press).

Robertson, D.S., Fallon, F.W., Carter, W.E. (1986).'Celestial Reference Coordinate Systems: Submilliarcsecond Precision Demonstrated.with VLBI Observations'. Astron. J. 91,6 .

Sovers, O.J. (1985). RSC (JPL) 85 R 02 (private communication).

Sovers, O.J. (1986). RSC (JPL) 86 R 02 (private communication).

Ryan, J.W., Ma, C. (1985). 'Crustal Dynamics Project Data Analysis', NASA Technical Memorandum 86229

Zhu, S.-Y., Mueller, I.I. (1983). 'Effects of Adopting new Precession, Nutation and Equinox Corrections on the Terrestrial Reference Frame'. Bull. Geod. 57, pp. 29-42. 


\section{DISCUSSION}

Johnston: Although the rotations between catalogs are small, the differences in declination can be quite large. This is demonstrated by the fact that these differences between JPL 86 and JPL83 vary from +5 mas at declination $-45^{\circ}$ to -5 mas at declination $70^{\circ}$. See the paper by deVegt \& Johnston, this volume.

T. Herring: Were there any single frequency data in the JPL-83 catalog which may explain the $\Delta \delta$ vs declination systematic behavior?

Reply by $\mathbf{R}$. Treuhaft: The catalog used in the $\Delta \delta$ vs. declination comparison was one of the last to include single-frequency data. Later catalogs, from roughly mid-1983 on do not include single-frequency data. When the $\Delta \delta$ vs. $\delta$ comparison was repeated at JPL with a dual-frequency catalog, 1983-4, the 10 mas effect vanished. There is another, apparently unrelated, $\Delta \delta$ vs $\delta$ effect at the 3 mas level, which arises when catalogs produced from VLBI data with different elevation-angle cuts are compared.

Ye Shu-hua: Can we assume that the NGS catalog is independent of the GSFC catalog? It seems they have some common sources of observations, and so the smaller differences between them may be expected.

Reply by Arias: The observations used for the implementation of the NGS catalog are a subset of those of the GSFC catalogs. In what concerns the rotation angles between NGS and GSFC catalogs, they are rather small for the case NGS-GSFC84, but this is not the case for GSFC86.

J. Hughes: Do you have the distribution of the 19 sources on the sky?

Reply by Arias: Only 3 of the 19 radio sources common to all the catalogs are in the Southern hemisphere, near the equator. The distribution in right ascension is not homogeneous. 